\title{
Women's experiences and preferences regarding breast imaging after completing breast cancer treatment
}

\author{
Susan Brandzel' \\ Dori E Rosenberg' \\ Dianne Johnson' \\ Mary Bush' \\ Karla Kerlikowske ${ }^{2-5}$ \\ Tracy Onega ${ }^{6,7}$ \\ Louise Henderson ${ }^{8}$ \\ Larissa Nekhlyudov 9,10 \\ Wendy DeMartini"l \\ Karen JWernli' \\ 'Group Health Research Institute, \\ Group Health Cooperative, Seattle, \\ WA, ${ }^{2}$ Department of Medicine, \\ ${ }^{3}$ Department of Epidemiology, \\ ${ }^{4}$ Department of Biostatistics, \\ ${ }^{5}$ Department of Veterans Affairs, \\ University of California, San Francisco, \\ San Francisco, CA, ${ }^{6}$ Department of \\ Biomedical Data Science, ${ }^{7}$ Department \\ of Epidemiology, Norris Cotton \\ Cancer Center, Geisel School of \\ Medicine at Dartmouth, Lebanon, \\ $\mathrm{NH},{ }^{8}$ Department of Radiology, \\ University of North Carolina, Chapel \\ Hill, NC, ${ }^{9}$ Department of Population \\ Medicine, Harvard Medical School, \\ ${ }^{10}$ Department of Medicine, Brigham \\ and Women's Hospital, Boston, MA, \\ "Department of Radiology, Stanford \\ University School of Medicine, \\ Stanford, CA, USA
}

This article was published in the following Dove Press journal:

Patient Preference and Adherence

I February 2017

Number of times this article has been viewed

Background: After treatment for breast cancer, most women receive an annual surveillance mammography to look for subsequent breast cancers. Supplemental breast MRI is sometimes used in addition to mammography despite the lack of clinical evidence for it. Breast imaging after cancer treatment is an emotionally charged experience, an important part of survivorship care, and a topic about which limited patient information exists. We assessed women's experiences and preferences about breast cancer surveillance imaging with the goal of determining where gaps in care and knowledge could be filled.

Participants and methods: We conducted six focus groups with a convenience sample of 41 women in California, North Carolina, and New Hampshire (USA). Participants were aged 38-75 years, had experienced stage $0-$ III breast cancer within the previous 5 years, and had completed initial treatment. We used inductive thematic analysis to identify key themes from verbatim transcripts.

Results: Women reported various types and frequencies of surveillance imaging and a range of surveillance imaging experiences and preferences. Many women experienced discomfort during breast imaging and anxiety related to the examination, primarily because they feared subsequent cancer detection. Women reported trust in their providers and relied on providers for imaging decision-making. However, women wanted more information about the treatment surveillance transition to improve their care.

Conclusion: There is significant opportunity in breast cancer survivorship care to improve women's understanding about breast cancer surveillance imaging and to provide enhanced support to them at the time their initial treatment ends and at the time of surveillance imaging examinations.

Keywords: breast cancer surveillance, mammography, breast MRI, cancer survivorship, oncology

\section{Introduction}

For women with a history of breast cancer, finishing initial treatment is a significant milestone; whether it involves the last radiation session, the final chemotherapy infusion, or surgery. The subsequent period of breast cancer care is known as the "surveillance period," a time when women see their care providers for follow-up visits and imaging to monitor for subsequent breast cancers.

According to national guidelines, women treated for breast cancer are recommended to receive annual mammograms in the absence of new signs or symptoms within 6 to 12 months after the completion of initial treatment and annually thereafter. ${ }^{1,2}$ While no formal guidelines recommend breast magnetic resonance imaging (MRI)
Correspondence: Susan Brandzel Group Health Research Institute, 1730 Minor Avenue, Suite 1600, Seattle, WA 98101 , USA

Tel +l 2062872380

Fax +I 2062872870

Email brandzel.s@ghc.org 
for breast cancer surveillance, some providers recommend breast MRI as an adjunct to mammography. ${ }^{3}$

Women's experiences with surveillance breast imaging are important to understand from both the patient and clinical perspectives. Carlos et $\mathrm{al}^{4}$ suggested that patient-centered outcomes in radiology should focus on a patient's experience of care beyond traditional measures of satisfaction. Specifically, they should include additional measures of benefits and harms, on outcomes relevant to women, and provide information to inform decision-making. Examining patient perspectives could help improve cancer survivorship planning and overall adherence to breast imaging recommendations.

Few studies have examined patient perspectives regarding imaging for cancer after completing treatment. In one prior study with adults who were diagnosed with various cancers, including early-stage breast cancer, participants reported high levels of gratitude for imaging tests that could be lifesaving; however, they often reported not understanding or receiving clear communication about the reasons for surveillance imaging from providers. ${ }^{5}$ In another study that included women without a history of breast cancer but who had experienced a false-positive result from breast imaging, more than half the participants reported being "scared" or "stressed" about mammography. ${ }^{6}$ We were unable to identify any studies focused on women's experiences or feelings about mammography or breast MRI used for surveillance imaging after completing the treatment for breast cancer. Given that there are $>2.9$ million breast cancer survivors in the US, ${ }^{7}$ there is a need to better understand and potentially improve women's perspectives about and experiences with surveillance imaging. Thus, we assessed women's experiences and preferences about breast cancer surveillance imaging with the goal of determining where gaps in care and knowledge could be filled by conducting a qualitative study of women with prior breast cancer in different geographic regions of the US.

\section{Materials and methods}

\section{Participant recruitment}

We conducted six focus groups among women in California, North Carolina, and New Hampshire, where there are Breast Cancer Surveillance Consortium (BCSC) registries with ongoing data collection systems. ${ }^{8}$ The BCSC is a collaborative network of breast imaging registries that began in 1994 with the goal of assessing the delivery and quality of breast imaging and related outcomes in the US. ${ }^{8}$ Each registry recruited women by mail and telephone to participate in the focus groups as a convenience-based sample either among those who had received a mammogram at facilities that contribute to that registry and had granted permission to be recontacted or through a local cancer registry.

Eligible women were aged $18-75$ years with a history of American Joint Committee on Cancer (AJCC) stage 0-III breast cancer diagnosed within the prior 5 years. Eligible women must have completed initial treatment for breast cancer (ie, surgery, chemotherapy, and/or radiation), but could be still taking adjuvant hormone therapy. Women were screened by telephone to ensure that they met the eligibility criteria. No individual risk factor or race/ethnicity data were collected. Two focus groups were conducted per geographic site. A mean number of seven women participated per group.

Institutional review board approval for this study was obtained from the following participating institutions: the University of California, San Francisco, the University of North Carolina, Chapel Hill, and Dartmouth College. All women provided written informed consent prior to focus group participation.

\section{Interviewing and data collection}

The six focus groups were led by the same facilitator who has $20+$ years of experience conducting research interviews and facilitating focus groups. The principal investigator and at least one patient partner from the study team also attended each focus group. The patient partners served as an important bridge to participants and were present to ease social discomfort. They also provided their impressions of the focus group content after the discussions, a pivotal contribution to the overall role that they played in this study. ${ }^{9}$ During the 2-hour discussions, we used a semistructured interview guide to ensure consistency but flexibility for each focus group. The guide, used to elicit participant's experiences and feelings about surveillance breast imaging, was developed from preparatory focus groups conducted as part of the grant development process and from input from patient partners, researchers, and clinicians on the study team. Verbatim written transcripts were generated by a confidentiality-bound stenographer present at each discussion.

\section{Analysis}

We used a combination of deductive and inductive thematic analysis ${ }^{10}$ to develop themes and subthemes that emerged from the discussions among our three-person coding team (SB, DER, and KJW). Coders had backgrounds in social and behavioral sciences and health services (SB), clinical psychology and health promotion (DER), and epidemiology and patient-involved research methods (KJW). The coders discussed emergent themes from initial individual reviews, 
which were used to develop a preliminary codebook and definitions. This codebook was used for the remaining four transcripts. Once all transcripts were coded, the team met to compare codes and reconcile discrepancies. Qualitative software Atlas.ti version 7.5.2 was used to organize final codes into themes.

\section{Results}

Forty-one women with prior breast cancer aged 38-75 years participated in the focus groups. Breast cancer stage at the time of diagnosis were stage $0: n=4(9.8 \%)$, stage $I: n=14$ (34.1\%), stage II: $n=18$ (43.9\%), stage III: $n=3$ (7.3\%), and unknown: $\mathrm{n}=2(<1 \%)$.

The discussion yielded feedback on the following four overarching themes: 1) type and frequency of surveillance breast imaging, 2) surveillance imaging experiences and preferences, 3) trust in providers, and 4) knowledge and decision-making about surveillance imaging.

\section{Theme I: type and frequency of surveillance breast imaging}

Women reported varied surveillance imaging patterns of mammography and breast MRI. Almost all women received mammography. Some women also received breast MRI. The most commonly reported pattern of surveillance breast imaging after completing breast cancer treatment was mammography every 3 or 6 months for 1 to 3 years after completion of treatment. Subsequently, most women reported having surveillance imaging either annually or every 6 months with a staggered pattern for those receiving mammography plus adjunct breast MRI. Most participants reported that either their oncologist or surgeon recommended and made the referrals for their imaging type and frequency after treatment. In a few cases, their primary care provider played this role.

\section{Theme 2: surveillance imaging experiences and preferences}

Women shared their imaging experiences and preferences, including being on a standardized imaging schedule, wanting the experience to be as painless as possible, and concern about subsequent breast cancers. Some women had either physically or emotionally negative breast imaging experiences. Many women experienced high levels of anxiety about and dislike for breast imaging, either because the test reminded them of their initial breast cancer diagnosis or because they feared having breast cancer again.

I dread it for a couple of weeks before. My anxiety builds as I get closer to the date.
I had to find the right dose for Ativan just to get me comfortable enough to get my boobies in there.

Another important aspect of the participants' experience focused on the discomfort of the imaging procedure. Some reported that radiology facilities actively tried to make them more comfortable either by being emotionally supportive or by providing warm blankets or pads to buffer the edge of the equipment. However, many women stated that mammography was painful or cold, or that staff were not empathetic. A few women had adverse reactions to the contrast dye injected during breast MRI. Others reported the procedure to be uncomfortable because of the position they had to hold or claustrophobia.

She puts this pink foam on the platform of the thing, and it just cushions everything.

You have to situate to the machine in order to get the image, and it's uncomfortable, and not only is it uncomfortable, it's dehumanizing.

Many women disliked the waiting period between completion of examination and receiving test results because the experience reminded them of when they were first evaluated for breast cancer. They said they felt stressed until they received their imaging results.

The worst point is between when you do your initial images and then you're waiting to see if you need more images. Because then as soon as they ask for more images, the mind just goes crazy about what they found and what they see.

She [provider] came in with her files close to her chest-is she holding it close to her chest because she's going to tell me something terrible?

Although some women were satisfied with the frequency of their imaging, others wanted imaging more often for reassurance that they did not have breast cancer again. A few women were concerned about receiving breast imaging too often because of radiation exposure from mammography or the invasiveness of imaging.

I feel good about my schedule.

If we had a safer imaging technique, I would get it twice, three times a year.

I'm not really educated about what kind of radiation you get from the mammographies and if it's something I should be concerned with. 
A portion of the discussion was dedicated to discussing experiences and feeling about false-positive and falsenegative results. Some women stated that they would prefer a false-positive result with follow-up procedures, such as a biopsy, and be reassured that they did not have another breast cancer than have a cancer be missed. However, other women wanted to avoid false-positive results and follow-up procedures because the additional tests caused too much worry, physical discomfort, and potential expense.

I would rather deal with the false positives than miss something, or think I might miss something.

I want something to be so definite right away and not to have any issues, no false positives, nothing. I want to know, and I want to know now, and I realize that's not possible.

Another important subtheme was the array of trust in the imaging modality. Some women whose breast cancer was not found with screening mammography had less trust in mammography. Other women were confident in mammography and did not feel the need for reassurance from additional imaging modalities.

I'm not totally sold about the mammograms, seeing how I had a perfectly normal mammogram four months before being diagnosed with breast cancer.

I'm now at the once a year both, every six months one. I feel like this is good. I feel good about continuing to get mammograms.

\section{Theme 3: trust in providers}

Many participants reported having complete trust in providers and therefore followed given recommendations. They felt their providers were sufficiently trained on the current recommendations for surveillance breast imaging. Some women mentioned that they sometimes asked questions about a provider's clinical choices, despite such inquiries being intimidating. A few women voiced distrust of provider motivations for their clinical decision-making regarding surveillance breast imaging, questioning whether decisions were being based on financial incentives rather than clinical impressions.

You have to trust your doctor. That's it. You have to trust that they know what they're doing.

I've learned over time that I have to ask her questions and my doctor, she's well known for the type I have, but she is so smart that I sometimes keep quiet and don't say anything.
And I realize now, no, no, no, I don't care how stupid I look, I'm going to ask her.

I've begged my oncologist, I'm like, 'I want an MRI.' And my oncologist says, 'The mammogram did its job. Your cancer was found on the mammogram. We will continue with surveillance with mammography, unless you have a problem.

\section{Theme 4: knowledge and decision-making about surveillance}

Most participants had never heard the term "surveillance" prior to the focus groups. Nonetheless, they remembered the time when their initial treatment, such as chemotherapy or radiation, ended. Some expressed that they were not given clear information about what follow-up care they would need in the short- or long-term future. Others had received a straightforward, detailed survivorship care plan.

To me it seems like there is a logistical handoff problem more knowledge is better. I would have, of course, liked a handout about that and everything else that I would need to know about.

I was told from the beginning that I would have to have yearly mammograms, just on the right side-they really make it very easy to do the whole follow-up.

Even with the offering of annual mammography, many of the women felt they did not have sufficient information to participate in decision-making about breast imaging after their treatment. Some women chose not to engage in imaging discussions with their providers. Women reported feeling confusion about the choices for surveillance imaging or about the frequency of imaging examinations. Other women reported more active involvement in decision-making about breast imaging, either because their provider initiated such discussions or because they actively sought it themselves.

Decision-making is really hard. You're presented with all of these things. And I kept saying I'm not a doctor, I don't know. I don't know. You know, what should I do? What is the right course?

My oncologist let me change to just mammograms every six months without the MRI. I like her manner. She says what she believes is the best, but then she doesn't argue or make you feel badly if you want to do something else, but the thing is, of course, she is the expert.

Finally, cost and insurance coverage was an important topic that sometimes affected participant preferences and 
experiences with surveillance imaging. Costs associated with copays or deductibles were a concern regarding surveillance breast imaging for some women, while for other women, cost was not a concern or barrier because she had insurance coverage or could afford to pay out of pocket costs.

The MRI scan-I didn't know was going to cost so much money. If I had known ahead of time, I might have made a different decision.

I would say that health care is really high on my list of priorities. When it comes to TV, I have limited basic. But health care. If I spend a couple of thousand dollars a year out of my pocket on health care, that's fine. I would rather do that than go to Puerto Vallarta for a week.

\section{Discussion}

This study validated that there is a wide range of radiology practices pertaining to breast cancer surveillance in the US, despite national guidelines recommending annual mammography. It also revealed that women experience the process of surveillance breast imaging in various positive and negative ways. Most importantly, it revealed areas in which patient care and clinical decision-making can be improved in order to be more educational and comfortable for women.

Several themes arose inductively that were not explicitly assessed via our interview guide. Many women expressed intense worry at the time of surveillance imaging because they feared having breast cancer again. The worries expressed by cancer survivors are well documented in prior literature. ${ }^{1-13}$ Schonberg et $\mathrm{al}^{14}$ determined that $44 \%$ of women with prior breast cancer experience anxiety about future mammograms. We believe that the pervasive, although not unanimous, acceptance of false-positive recall and high anxiety associated with imaging examinations were indicators that women might be overestimating their risk of cancer second breast cancer events. Fardell et $\mathrm{al}^{15}$ highlight the dearth of information available to women about their risk of subsequent breast cancers, calling for improved tools to manage this fear.

Women reported high levels of provider trust and did not often question their imaging recommendations. It was not clear whether providers were recommending imaging regimens based on clinical guidelines, clinical autonomy, or institutional policy. This topic warrants further exploration. Given the various reported experiences and recommendations, clarifying justification for imaging recommendations will likely help to alleviate some women's fear and increase understanding of surveillance breast imaging modalities.
Radiology facilities have the opportunity to improve breast imaging experiences for all women, not just women with prior breast cancer. Women reported wanting providers and facilities to make mammography and breast MRI more physically and emotionally comfortable including same day reporting of results within a short time, especially in light of their breast cancer history. One study found that women had a better experience with breast MRI than with mammography but also emphasized the importance of the facility's sensitivity to women's needs and their experience conducting the imaging as being a high priority regardless of imaging modality. ${ }^{16}$

While our study adds to the literature and has considerable strengths, such as the geographically diverse sample with various cancer stages, it has limitations. First, the women were all insured, able to attend a weekday focus group, and were not proportionately racially diverse to the US population. Therefore, we may not have obtained key issues related to health care decision-making and access in underserved and underrepresented populations. Second, we did not collect information regarding breast cancer risk factors, including genetic mutations, family history, or breast density, elements that could have influenced surveillance preferences and experiences. Third, we included a highly heterogeneous sample by age at diagnosis and stage of breast cancer. These two factors can drive clinical decision-making about surveillance imaging and thus may explain some of the deviation we heard regarding adherence to surveillance imaging guidelines. Despite these limitations, our study provides important new information previously lacking in the literature. There is significant opportunity in breast cancer survivorship care to improve women's understanding about breast cancer surveillance imaging, to provide enhanced psychological support at the time their initial treatment ends and continue that support at the time of surveillance imaging examinations.

\section{Conclusion}

There is scant research that has examined women's perspectives about breast cancer surveillance imaging. Our results suggest that women treated for stage 0 -III breast cancer within 5 years of diagnosis have diverse experiences and feelings regarding surveillance breast imaging. Due to the large number of breast cancer survivors who receive annual surveillance mammograms in the US, improving processes to more robustly support the psychological and physical experience of breast cancer surveillance imaging is important for survivorship care. 
Our study also reveals that, although annual surveillance mammography is the guideline standard, ${ }^{2}$ some women undergo adjunct surveillance breast MRI too. Additionally, many women in our study received surveillance mammography more often than annually following treatment despite the absence of signs or symptoms. These reported surveillance patterns suggest an opportunity in clinical care to either better align imaging practices with national guidelines or inform women about reasons for deviations from guidelines.

\section{Acknowledgments}

We would like to express our sincere gratitude to the study participants from these focus groups. We also wish to thank Dr Diana Buist for her guidance and study coordinators Jennette Sison (UCSF), Katie Marsh (UNC), and Martha Goodrich (Dartmouth). Research reported in this publication was funded through a Patient-Centered Outcomes Research Institute (PCORI) Award (CE-1304-6656). The views presented in this publication are solely the responsibility of the authors and do not necessarily represent the views of the Patient Centered Outcomes Research Institute (PCORI), its Board of Governors or Methodology Committee.

\section{Disclosure}

The authors report no conflicts of interest in this work.

\section{References}

1. Khatcheressian JL, Hurley P, Bantug E, et al. American Society of Clinical Oncology. Breast cancer follow-up and management after primary treatment: American Society of Clinical Oncology clinical practice guideline update. J Clin Oncol. 2013;31(7):961-965.

2. National Comprehensive Cancer Network: NCCN Guidelines. Fort Worth, PA: NCCN; 2013 [cited August 26, 2015]. Available from: http:// www.nccn.org/professionals/physician_gls/f_guidelines.asp. Accessed September 13, 2016.
3. Brennan S, Liberman L, Dershaw DD, Morris E. Breast MRI screening of women with a personal history of breast cancer. AJR Am J Roentgenol. 2010;195(2):510-516.

4. Carlos RC, Buist DS, Wernli KJ, Swan JS. Patient-centered outcomes in imaging: quantifying value. $J$ Am Coll Radiol. 2012;9(10):725-728.

5. Thornton RH, Dauer LT, Shuk E, et al. Patient perspectives and preferences for communication of medical imaging risks in a cancer care setting. Radiology. 2015;275(2):545-552.

6. Harvey S, Gallagher AM, Nolan M, Hughes CM. Listening to women: expectations and experiences in breast imaging. $J$ Womens Health (Larchmt). 2015;24(9):777-783.

7. Howlader N, Noone AM, Krapcho M, et al. SEER Cancer Statistics Review, 1975-2012. Bethesda, MD: National Cancer Institute; based on November 2014 SEER data submission, posted to the SEER web site, April 2015 [updated August 20, 2015; cited September 20, 2015]. Available from: http://seer.cancer.gov/csr/1975_2012/. Accessed September 13, 2016.

8. Ballard-Barbash R, Taplin SH, Yankaskas BC, et al. Breast Cancer Surveillance Consortium: a national mammography screening and outcomes database. AJR Am J Roentgenol. 1997;169(4):1001-1008.

9. Johnson DSBM, Brandzel S, Wernli KJ. The patient voice in research - evolution of a role. Res Involvem Engagem. 2016;2:6.

10. Boyatzis RE. Transforming Qualitative Information: Thematic Analysis and Code Development. Thousand Oaks: SAGE Publications; 1998.

11. Tewari A, Chagpar AB. Worry about breast cancer recurrence: a population-based analysis. Am Surg. 2014;80(7):640-645.

12. Janz NK, Friese CR, Li Y, Graff JJ, Hamilton AS, Hawley ST. Emotional well-being years post-treatment for breast cancer: prospective, multi-ethnic, and population-based analysis. J Cancer Surviv. 2014; 8(1):131-142.

13. De Vries J, Den Oudsten BL, Jacobs PM, Roukema JA. How breast cancer survivors cope with fear of recurrence: a focus group study. Support Care Cancer. 2014;22(3):705-712.

14. Schonberg MA, Silliman RA, Ngo LH, et al. Older women's experience with a benign breast biopsy - a mixed methods study. J Gen Intern Med. 2014;12:1631-1640.

15. Fardell JE, Thewes B, Turner J, et al. Fear of cancer recurrence: a theoretical review and novel cognitive processing formulation. $J$ Cancer Surviv. 2016;10(4):663-673.

16. Bredart A, Kop JL, Fall M, et al. Magnetic Resonance Imaging Study Group. Perception of care and experience of examination in women at risk of breast cancer undergoing intensive surveillance by standard imaging with or without MRI. Patient Educ Couns. 2012;86(3): 405-413.
Patient Preference and Adherence

\section{Publish your work in this journal}

Patient Preference and Adherence is an international, peer-reviewed, open access journal that focuses on the growing importance of patient preference and adherence throughout the therapeutic continuum. Patient satisfaction, acceptability, quality of life, compliance, persistence and their role in developing new therapeutic modalities and compounds to optimize

\section{Dovepress}

clinical outcomes for existing disease states are major areas of interest for the journal. This journal has been accepted for indexing on PubMed Central. The manuscript management system is completely online and includes a very quick and fair peer-review system, which is all easy to use. Visit http://www. dovepress.com/testimonials.php to read real quotes from published authors. 